\title{
Simultaneous dislocation of the Lisfranc and Chopart joints: A case report, surgical technique, and literature review
}

\author{
Rodolfo Morales-Avalos*, Ángel Arnaud-Franco, Luis E. Martínez-Manautou, \\ Raymundo A. Rodríguez-Torres, Héctor Noé G. Silva-Ramos, and Víctor M. Peña-Martínez \\ Foot and Ankle Module, Orthopedics and Traumatology Service, School of Medicine and "Dr. Jose Eleuterio González" University Hospital, \\ Universidad Autónoma de Nuevo León, Monterrey, Nuevo León, Mexico
}

\begin{abstract}
Introduction: Simultaneous luxation of the Lisfranc and Chopart joints is rare and presents high morbidity. There is not enough information in the literature regarding the treatment of these lesions. Clinical Case: A 30-year-old male diagnosed with a simultaneous dislocation of the Lisfranc and Chopart joints who underwent a closed reduction and osteosynthesis with 3.5 $\mathrm{mm}$ screws and Kirschner nails. Conclusion: The early open reduction and internal fixation led to an adequate clinical result with a satisfactory evolution, which is consistent with what has been published in the literature.
\end{abstract}

Key words: Chopart joint. Lisfranc joint. Fracture dislocation. Combined dislocation. Simultaneous dislocation. Mid-foot.

\section{Introduction}

Mid-foot is defined as the anatomical region between the Chopart joint (comprised the calcaneocuboid and astragalus scaffold joints) and the Lisfranc joint (comprised the three cuneiform bones on the medial side and the two metatarsal cuboid bones on the lateral side). Both joints are considered a part of the mid-foot and fulfill biomechanical functions linked to the foot's stability and motility ${ }^{1}$. Fractures and luxations of the mid-foot are rare, representing between 0.1 and $0.9 \%$ of all body fractures. Main and Jowet proposed a classification for mid-foot fractures based on their production mechanism, with the type- 5 fractures resulting from crushing caused by random force of great magnitude and speed ${ }^{2}$. Among mid-foot trauma injuries, injuries as a result of crushing represent $5-6 \%$ of them, with a combined luxation of the Chopart and Lisfranc joints the rarest and most severe of all ${ }^{1}$, with a low prevalence? ${ }^{2}$.

Mid-foot injuries can occur in up to $10 \%$ of multiple trauma patients who suffered ankle or foot lesions due to driving accidents, frequently going unnoticed, or not fully diagnosed during the patient's initial assessment ${ }^{3}$. Sancho-Gonzalez described that most of these injuries have a poor functional prognosis and typically result in a high degree of morbidity in the long run and even loss of the affected limb ${ }^{4}$.

Thus far, there are $<50$ reported cases in the literature, with varied treatments, and without an established therapeutic algorithm ${ }^{4}$. We present a case of simultaneous luxation of the Chopart and Lisfranc joints in a

\section{Correspondence:}

${ }^{*}$ Rodolfo Morales-Avalos

E-mail: rodolfot59@ hotmail.com
Available online: 18-03-2021

Date of reception: 14-08-2020

Date of acceptance: $27-10-2020$

DOI: 10.24875/RMU.20000073
Medicina Universitaria. 2021;23(1):24-28 www.medicinauniversitaria.org

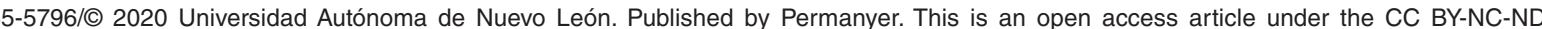
license (http://creativecommons.org/licenses/by-nc-nd/4.0/). 
30-year-old male, which occurred after an automobile accident, surgical management, clinical follow-up at 2 years, and a review of the existent literature.

\section{Case presentation}

A 30-year-old male, without relevant medical history, arrived at the emergency room after participating in a motorcycle accident an hour previous to his arrival. During the initial examination, he presented generalized edema and deformity of his right foot (Fig. 1), inability to hold his own weight and walk around, as well as limited ankle mobility. The right foot was cold and pale compared to its counterpart. Pedis and tibial pulses were present but diminished, capillary refill time was $3 \mathrm{~s}$, and he did not present sensitive alterations. The patient's foot skin did not present wounds but had a visible hematoma on the right foot's bottom. Tibial-talar mobility was normal but painful, and initial X-rays revealed a luxation of the astragalus navicular component at the transverse tarsal joint level, with medial and dorsal displacement of the astragalus and a residual calcaneocuboid articular congruency. An inadequate alignment at the expense of both lateral and dorsal metatarsal displacements was identified at a tarsometatarsal joint level, as well as an opening of articular space between the medial and intermedial wedges, concerning a Lisfranc fracture dislocation, classified as a type-A Myerson (full lateral incongruence) linked to an oblique fracture of the base of the second metatarsal and the lateral wedge (Fig. 2).

During initial treatment in the ER, a close reduction of the Chopart joint was performed with the objective of limiting the edema, conducted with the patient under sedation, clinically observing an improvement in pain and the foot perfusion presenting a coloring and temperature similar to that of the collateral and pedis pulse and posterior tibial present. Subsequently, the patient underwent a fine slice computerized axial tomography (CAT scan) and a Doppler vascular ultrasound on the right lower limb. The reduction of the astragalonavicular luxation was identified in the fine slice CAT scan, providing evidence of an intra-articular bone fragment of $1.8 \times 4.5 \mathrm{~mm}$. Moreover, a partial reduction of the Lisfranc luxation was identified, finding a correct alignment in the first metatarsal and the medial cuneiform bone, leading to the lateral displacement of the second and third metatarsals (Fig. 3). The Doppler ultrasound revealed a present and triphasic blood flow posterior to the reduction in the pedis arteries and posterior tibial.

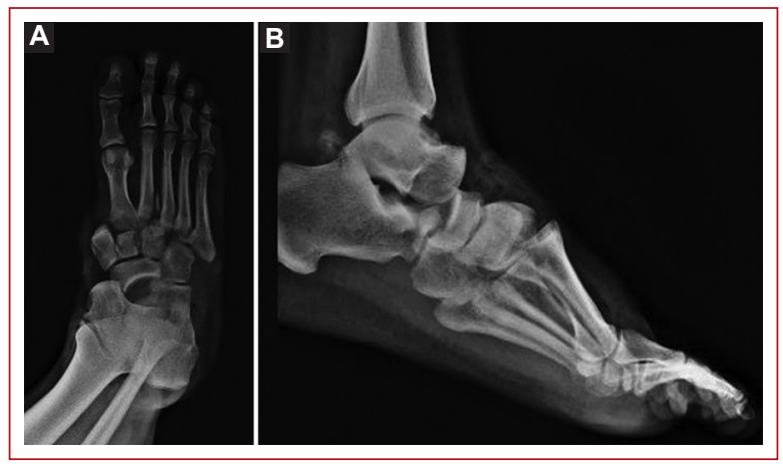

Figure 1. Clinical image of the patient during his hospital admission. A: lateral view. B: medial view.

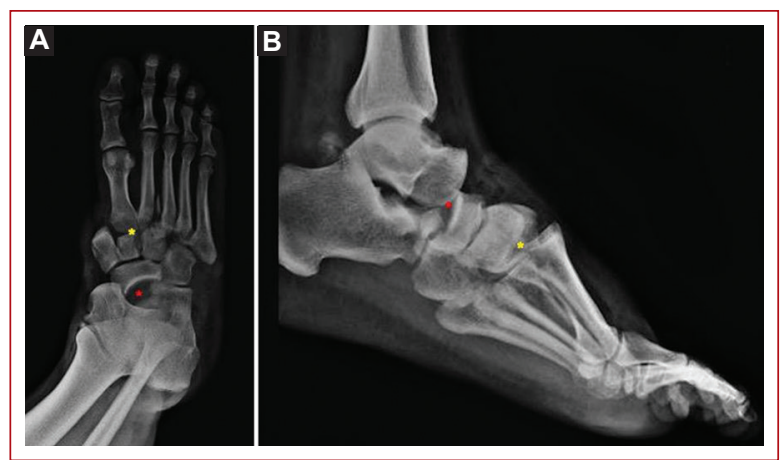

Figure 2. Initial X-rays where a simultaneous luxation of the Lisfranc (yellow asterisk) and Chopart (red asterisk) joint is evident. A: oblique projection. B: lateral projection.

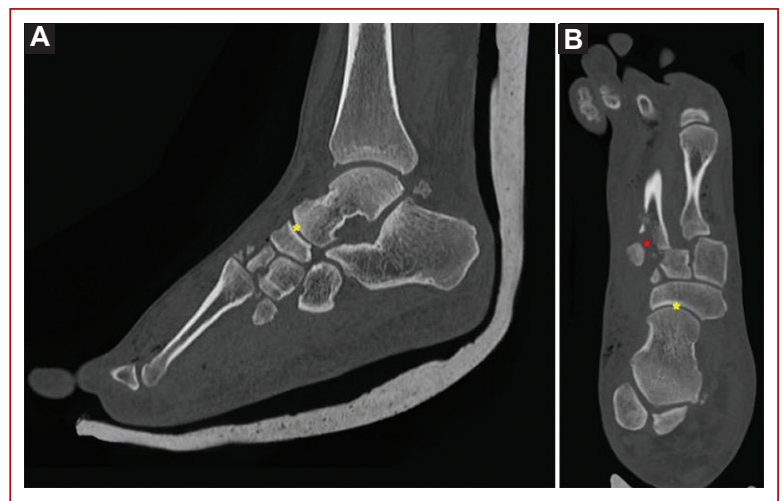

Figure 3. CAT scan taken posterior to initial closed reduction. A: sagittal slice where we can observe a correct reduction of the Chopart joint (yellow asterisk). B: sagittal slice where we are able to observe the fracture of the base of the second metatarsal (red asterisk) and a good reduction of the astragalus scaphoid joint (yellow asterisk). 


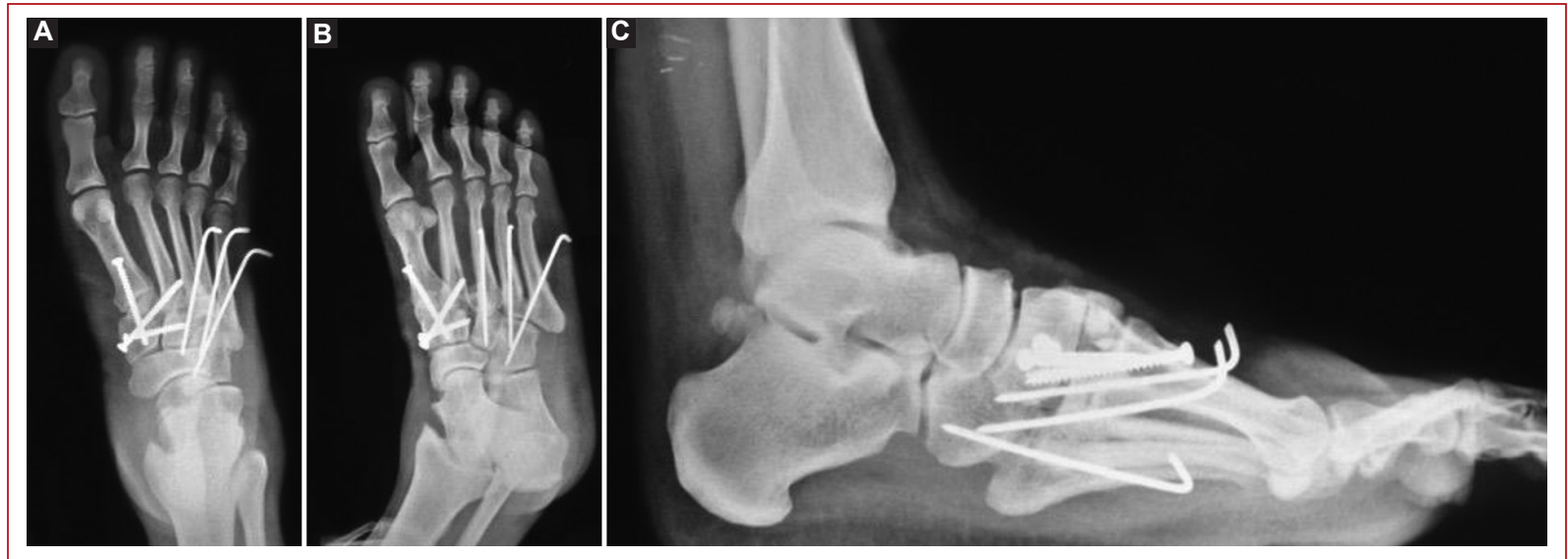

Figure 4. Immediate post-surgical X-rays where we are able to observe an adequate reduction of both joints with the material of osteosynthesis. A: anteroposterior projection. B: oblique projection. C: lateral projection.

The patient was brought into surgery urgently, performing an open reduction and internal fixation (ORIF) of the Lisfranc joint under the fluoroscopic guide.

The patient was placed in the supine position with a pneumatic tourniquet. Two dorsal incisions were performed longitudinally over the Lisfranc joint to adequately reduce the metatarsals (starting with the second metatarsal); intra-articular fragments were removed. A correct articular congruence was observed. For fixation, three all-thread screws $(3.5 \mathrm{~mm})$ were used. The first one was placed in a medial to lateral direction from the medial cuneiform bone to the base of the second metatarsal, the second screw was introduced in the same direction from the medial cuneiform bone toward the intermedial cuneiform bone percutaneously, and the last one was placed in an anteroposterior direction from the first metatarsal to the medial cuneiform bone. Subsequently, 3 Kirschner \#0.62 nails were placed to fix the third, fourth, and fifth metatarsals percutaneously (the first nail was introduced up to the lateral cuneiform bone and the other two up to the cuboid bone) (Fig. 4).

The stability of the Chopart joint was assessed intraoperatively under fluoroscopic control, and it was decided not to fix it due to the adequate stability it presented. It was covered with a Jones dressing postoperatively, and the patient was put under hospital surveillance for 3 days. After 2 weeks, the suture was removed and a rigid boot was placed for 8 weeks without allowing any pressure on the foot. Eight weeks after the operation, the nails were removed, and the patient started progressive support using a rigid boot. After 12 weeks, the boot was removed to begin mobility and

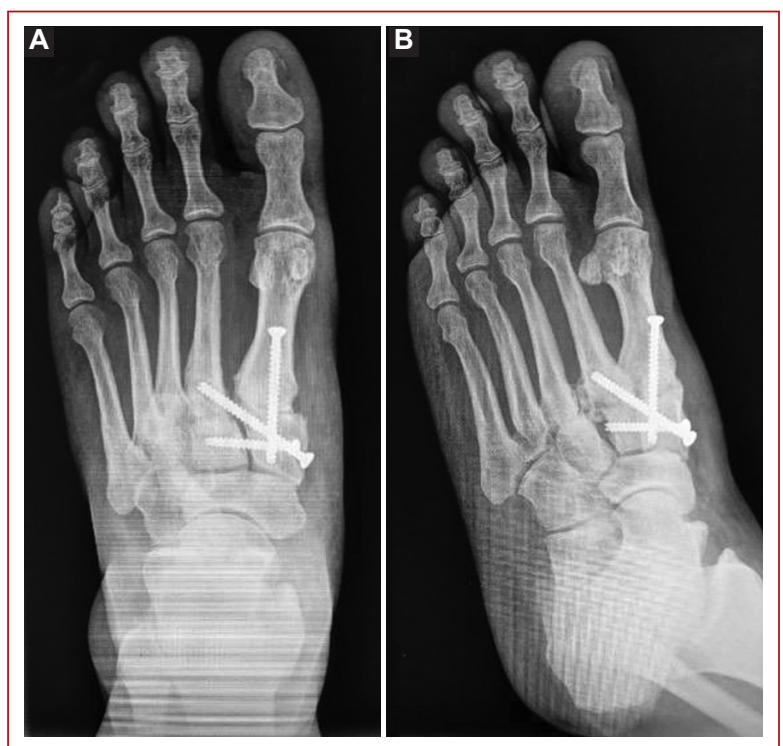

Figure 5. A-B: radiographic follow-up at 2 years of the patient.

rehabilitation therapy. At radiological follow-up at 3 months, 6 months, and 2 years (sic), we did not observe a loss in the reduction and mild arthrosis data (Fig. 5). The patient was monitored for 2 years with the application of the American Orthopaedic Foot and Ankle Society (AOFAS) scales, with a score of 63 at 3 months and 84 at 6 months, ending up with a score of 94 in the applied scale at 2 years. The patient had a favorable evolution, not referencing pain when resting or walking, having articular stability assessed by objective and subjective methods, and currently reinstated to his sporting and work activities. 


\section{Discussion}

Mid-foot injuries are rare since the area is comprised joints with strong ligamentous structures that typically resist rupture ${ }^{5}$. The main causes of mid-foot luxation are driving accidents and falls from a great height. In a revision which included 155 patients, $72 \%$ of the midfoot injuries were caused by traffic accidents, of which $55 \%$ were accidents while in automobile and $17 \%$ were while riding a motorcycle ${ }^{1}$, as in our case.

Injuries with a combined luxation of the Lisfranc and Chopart joints are frequently part of the so-called "crushing injuries" of the foot, characterized by an extensive injury of soft tissues, high rates of deep infection, and development of compartmental syndrome. These crushing injuries lead to the greatest degree of residual deterioration of all the foot injuries ${ }^{6}$. However, even in these types of injuries, it has been determined that an open reduction with the anatomical restoration of the articular surfaces combined with a stable internal fixation improves the final clinical result of the patient ${ }^{1}$.

Ritcher et al. analyzed injuries of the mid-foot over a period of 25 years, concentrating on the production mechanism, the time elapsed from the time of the injury to its treatment, and the utilized treatment method, evaluating them through clinical and radiographic examinations with at least a year of follow-up (mean of 9 years, range between 1.3 and 25 years), concluding that the injury where there were the worst functional results due to the development of post-traumatic arthritis, the onset of complex regional pain syndrome, sensory deficit, and cutaneous necrosis, was the combined injury of the Lisfranc and Chopart joints. Similarly, this injury represented the highest cause of amputations needed, deep infections, reinterventions, performance of fasciotomies, and primary arthrodesis performance as definitive treatment compared to all other types of injuries ${ }^{1}$. In the case of our patient, at 2 years of follow-up, none of these complications occurred.

Lu et al. proposed a classification of Lisfranc injuries based on a retrospective analysis of 5 years, including 54 isolated or combined Lisfranc injuries, classifying them into three main types. Establishing type three as a "Lisfranc ligament injury combined with a luxation or fracture of the Chopart joint," and reporting five cases of it $(9.25 \%)$, all cases were treated with a bridging plate and during follow-up at 1 year obtained an average value on the AOFAS scale of 79.7 points $^{6}$. In our study, the patient at 1 year presented a score of 82 in the AOFAS scale at a year of follow up and 94 at 2-year follow-up.
There is little information about the clinical results of the different methods of treatment employed. However, the main purpose of treatment is to reestablish the correct orientation of the foot through an early anatomical reduction ${ }^{7}$. Previous reports have determined that the key to reduction is the second metatarsal, which should be aligned at the beginning of surgery since the rest of the metatarsals will not be reduced unless the second metatarsal is properly reduced ${ }^{1}$. Concerning the fixation method, the use of cannulated or conventional screws over Kirschner nails and bridging plaques has been suggested due to its lower rate of reduction loss and surgical wound dehiscence and a greater possibility of early ambulation since it does not restrict articular mobility as much compared to the bridging plaques ${ }^{8}$. Nevertheless, other authors defend the use of bridging plaques because of their possibility to maintain bone longitude and ability to stabilize small fragments that are non-candidates for individual fixation ${ }^{5}$. In our case, we decided to use an open reduction to accomplish proper anatomical congruence, with the possibility to perform a less invasive approach when placing the compression screws and percutaneous nails, since we considered their use would allow for less manipulation of soft tissues and early ambulation.

Contraindications of performing an open reduction and internal fixation include the presence of active superficial infection or poor conditions of the soft tissues, advanced peripheral vascular disease, chronic venous insufficiency with the presence of ulcers, Charcot atrophy, and non-cooperative patients. In these cases, the use of a closed reduction ought to be performed as anatomically as possible ${ }^{7}$. In massive generalized edema without compartment syndrome, the surgery should be postponed until the edema has decreased. In said cases, a closed reduction of the evident deformities ought to be performed, immobilized with a short posterior brace, elevation of the limb, application of ice, and administration of intravenous nonsteroidal anti-inflammatories up to the surgery. Primary arthrodesis is only indicated in massive and irreversible articular damage with a high degree of possibility of the development of post-traumatic arthrosis ${ }^{9}$. Primary or secondary amputation is reserved for cases of uncontrolled deep infection with a risk of sepsis, irreparable vascular injuries, or an extensive loss and contamination of soft tissues in the context of a patient with multiple ipsilateral fractures of the lower limb ${ }^{9}$. In table 1, there is a presentation of published cases to date.

In the case of significant shortening of the medial or lateral columns, extensive articular comminution, 
Table 1. Comparative table of the different reports or series of published cases to date

\begin{tabular}{|l|l|l|l|l|}
\hline Author (year) & $\begin{array}{l}\text { Number of } \\
\text { cases }\end{array}$ & Production mechanism & Treatment & Complications \\
\hline Abdelgaid et al., 2013 & 2 & Automobile accident & RAFI & No \\
\hline Richter et al., 2004 & 22 & $\begin{array}{l}\text { Great height fall }(n=10) \\
\text { Automobile accident }(n=9) \\
\text { Fall of heavy object }(n=2)\end{array}$ & $\begin{array}{l}\text { Primary amputation }(n=9) \\
\text { Closed reduction and } \\
\text { internal fixation }(n=2)\end{array}$ & $\begin{array}{l}\text { Compartment síndrome }(n=2) \\
\text { Conversion to amputation }(n=2) \\
\text { Deep infection }(n=3)\end{array}$ \\
\hline Lu et al., 2017 & 5 & Automobile accident & RAFI & Reep infection $(n=2)$ \\
\hline
\end{tabular}

soft tissue in poor condition, or patients who require additional surgeries in the thoracic, cranial, or abdominal cavities, the use of external fixation is recommended ${ }^{10}$.

\section{Conclusion}

We conclude that simultaneous luxations of both the Lisfranc and Chopart joints are a rare pathological entity, one which comes with significant functional sequelae. Therefore, it ought to be treated surgically early, always looking for an anatomical reduction of articular surfaces and the reestablishment of the longitude of both lateral and medial columns of the foot. Ruling out the presence or development of a compartment syndrome is a must, following an adequate physical rehabilitation to reduce long-term functional sequelae. In our patient's case, early open reduction and internal fixation led to an adequate clinical result with a satisfactory evolution, which concurs with what has been published in the literature.

\section{Acknowledgments}

To Dr. Ernesto C. Martínez Ávila for the help provided in the technical review of this article.

\section{Conflicts of interest}

The authors declared not to have any conflicts of interest.

\section{Funding}

This study was financed by the Orthopedics and Traumatology Service's resources.

\section{Ethical disclosures}

Protection of human and animal subjects. The authors declare that the procedures followed were in accordance with the regulations of the relevant clinical research ethics committee and with those of the Code of Ethics of the World Medical Association (Declaration of Helsinki).

Confidentiality of data. The authors declare that they have followed the protocols of their work center on the publication of patient data.

Right to privacy and informed consent. The authors have obtained the written informed consent of the patients or subjects mentioned in the article. The corresponding author is in possession of this document.

\section{References}

1. Richter M, Wippermann B, Krettek C, Schratt HE, Hufner T, Therman H. Fractures and fracture dislocations of the midfoot: occurrence, causes and long-term results. Foot Ankle Int. 2001;22:392-8.

2. Main BJ, Jowett RL. Injuries of the midtarsal joint. J Bone Joint Surg Br. 1975;57:89-97.

3. Benirschke SK, Meinberg E, Anderson SA, Jones CB, Cole PA. Fractures and dislocations of the midfoot: lisfranc and chopart injuries. J Bone Joint Surg Am. 2012;94:1325-37.

4. Sancho-González I, Menéndez-García M. Missed chopart dislocation. The importance of being aware of midtarsal injuries. An Sist Sanit Navar. 2016;39:153-8.

5. Mansour A, Fallat L. Temporary bridge plating of the medial column in chopart and lisfranc injuries. Foot Ankle Online J. 2017;10:5-10.

6. Lu J, Ren W, Li N, Holledge MM, Hu Y. Assessment of the typology of lisfranc injuries of the foot. J Surg. 2017;2017:1-10.

7. Abdelgaid SM, Salah M, Abdulsalam S, Abdulmalak F. Closed reduction and percutaneous fixation of lisfranc joints injuries: possibility, technique and results. Clin Res Foot Ankle. 2013;1:109.

8. Richter M, Thermann $\mathrm{H}$, Huefner $\mathrm{T}$, Schmidt $U$, Goesling $T$, Krettek $\mathrm{C}$. Chopart joint fracture-dislocation: initial open reduction provides better outcome than closed reduction. Foot Ankle Int. 2004;25:340-8.

9. Richter M, Thermann H, Hüfner T, Schmidt U, Krettek C. Aetiology, treatment and outcome in lisfranc joint dislocations and fracture dislocations. Foot Ankle Surg. 2002;8:21-32.

10. Klaue K. Treatment of chopart fracture-dislocations. Eur J Trauma Emerg Surg. 2010;36:191-5. 